\title{
PRODUTIVIDADE E CUSTOS DE EXTRAÇÃO DE MADEIRA DE EUCALIPTO COM CLAMBUNK SKIDDER ${ }^{1}$
}

\author{
Pedro Henrique Alves dos Santos², Amaury Paulo de Souza ${ }^{3}$, Felipe Leitão da Cunha Marzano ${ }^{4}$ e \\ Luciano José Minette ${ }^{3}$
}

\begin{abstract}
RESUMO - Os clambunk skidders, utilizados na extração de madeira de eucalipto no Brasil, são máquinas complexas, de alta tecnologia e de alto valor de aquisiçao. Os objetivos da pesquisa foram analisar a produtividade, a eficiência operacional e os custos operacionais e de produção desse trator florestal. Esta pesquisa foi desenvolvida em povoamentos de eucalipto (hibrido de Eucalyptus urophylla $\mathrm{x}$ Eucalyptus grandis) de uma empresa florestal no Estado de São Paulo. O sistema de colheita de madeira foi o de árvores inteiras com o processamento das árvores em forma de cavacos, em pátios localizados às margens dos talhões. A metodologia utilizada na avaliação da produtividade foi constituída de técnicas de estudos de tempos e movimentos. Na avaliação dos custos foram determinados os custos operacionais e os de produção. Os resultados indicaram que as atividades que consumiram maior parte do tempo do ciclo de trabalho foram o carregamento e a viagem com carga. A produtividade foi significativamente reduzida com o aumento da distância de extração. A análise de custos operacionais indicou que os custos variáveis foram os de maior valor, seguidos pelos custos fixos e de administração. O custo de extração do clambunk aumentou com a distância de extração, pois essa variável afetou os tempos de viagem. A diminuição desse custo seria possível pela reorganização do processo de trabalho com base na distância e nos outros fatores estudados.
\end{abstract}

Palavras-chave: Colheita florestal; Trator florestal arrastador; Tempo gasto.

\section{PRODUCTIVITY AND COSTS OF EUCALYPTUS WOOD EXTRACTION WITH CLAMBUNK SKIDDER}

\begin{abstract}
The efficient planning and control of timber harvesting operations are essential to increase productivity and reduce costs. The research objectives were to evaluate the time consumed by the elements of the work cycle, productivity, cost and operational efficiency of a Clambunk Skidder. The research was carried out in a stand of eucalyptus, in a Forest Company, São Paulo State, Brazil. The full-tree harvesting system was utilized and the trees were processed into chips, in landings located at roadside. The methodology used in assessing the productivity consisted of consolidated techniques of motion and time studies that include systematic analysis of the production process including time consumption, production, influential factors, degree of mechanical availability and efficiency. In costs assessment were determined operating costs, fixed costs and variable costs. Elements of the work cycle which most time consuming were the Loading and Travel loaded. The productivity was significantly influenced by the extraction distance. The operational cost analysis indicated that the most important, in descending order, were the variable, the fixed and the administration costs. The Clambunk production cost varied with extraction distance, because this variable affected the travelling times. A reduction in production cost could be possible by reorganizing the work process based on distance and other factors studied.
\end{abstract}

Keywords: Timber harvesting; Tractor skidding; Time study.

\footnotetext{
${ }^{1}$ Recebido em 27.07.2009 aceito para publicação em 12.05.2013.

${ }^{2}$ Programa de Iniciação Científca do CNPq pela Universidade Federal de Viçosa, UFV, Brasil. E-mail: <alves.santos.p@ gmail.com>.

${ }^{3}$ Departamento de Engenharia Florestal da Universidade Federal de Viçosa, UFV, Brasil.E-mail: <amaury@ufv.br $>$ e $<$ minette@ufv.br>.

${ }^{4}$ Programa de Pós-Graduação em Ciência Florestal pela Universidade Federal de Viçosa, UFV, Brasil. E-mail: <felipe.marzano@ufv.br>.
} 


\section{INTRODUÇÃO}

Como resultado do crescimento da economia nacional e da competição resultante da globalização, a partir de 1990 o setor florestal passou por várias mudanças. Uma delas foi a introdução de modernas máquinas e equipamentos para atender ao aumento da demanda por produtos florestais e às exigências do mercado globalizado. Esse incremento tecnológico valorizou o grau de competitividade das empresas, conferindo maior importância ao planejamento dos processos produtivos. Os custos das operações de extração de madeira têm grande influência na composição dos custos totais de produção florestal, chegando a representar mais de $50 \%$ do custo final da madeira (MACHADO, 1989). Por isso, o eficiente planejamento e controle das operações de extração são essenciais para a racionalização do trabalho, redução dos custos, aumento da qualidade do produto e diminuição dos danos ambientais. Os estudos dos tempos e movimentos têm sido muito utilizados para avaliar a produtividade e para planejar e racionalizar sistemas de extração florestal (WESTER, 2003; ADEBAYO, 2007; GOYCHUK, 2011).

A tendência no sentido de aumento da mecanização é atribuída à necessidade de se preocupar com a produtividade, segurança, questões ambientais e custos. Um fluxo constante de madeira durante todo o ano é imperativo para gerar receitas, cobrir custos e obter lucros (LeDOUX, 2010).

O clambunk skidder é uma máquina destinada ao arraste de feixes de toras compridas ou árvores inteiras da área de corte até o local de processamento. O carregamento é realizado por uma grua, que coloca toras ou árvores inteiras sobre uma garra invertida apoiada no chassi do trator (MACHADO et al., 2008). Essa máquina tem capacidade para extrair maior volume do que os skidders convencionais. E isso a permite operar em maiores distâncias de extração, podendo contribuir para diminuir a densidade de estradas florestais. Sua eficiência aumenta quando um feller-buncher é utilizado para formar feixes de madeira de forma a facilitar a operação de carregamento.

O sistema de colheita de árvores inteiras utilizando clambunk skidder para extração de madeira e seu processamento em cavacos em pátios localizados às margens dos talhões é pouco estudado. Nesse caso, a utilização do clambunk permite extrair, por ciclo, grande volume de madeira com galhos e copas. Essa extração é possível por causa da capacidade de carga da máquina e de o feller-buncher abater as árvores e formar feixes, facilitando o carregamento de grandes volumes em menor espaço de tempo

O estudo de tempos e movimentos auxilia na análise do trabalho, com a finalidade de criar ou alterar sistemas de colheita para aumentar a eficiência e a produtividade e diminuir custos (VALVERDE, 1996; NURMINEN et al., 2006).

Os principais fatores que afetam a produtividade são a distância de extração, a densidade do talhão, o volume de madeira por árvore e a topografia. Vários autores concluíram que a distância de extração afeta a produtividade devido ao tempo consumido no ciclo de trabalho. Porém, há necessidade de estudar esse efeito para casos específicos como o do sistema adotado, em que a madeira é extraída e transformada em cavaco em fluxo contínuo. Esse fluxo requer que o volume de extração seja compatível com a capacidade de processamento do picador.

Devido ao elevado custo relacionado a aquisição, manutenção e operação do clambunk, são necessários estudos que auxiliem na otimização de seu uso. Em sistemas constituídos por mais de uma máquina devem ser identificados os gargalos e os melhores meios de superá-los.

O objetivo desta pesquisa foi analisar a produtividade, a eficiência operacional e os custos operacionais e de produção do clambunk skidder.

\section{MATERIAL E MÉTODOS}

\subsection{Local da pesquisa}

Esta pesquisa foi desenvolvida no Município de Capão Bonito, Estado de São Paulo, em áreas de operação de colheita florestal pertencentes a uma empresa do setor de celulose e papel.

O relevo da área de coleta de dados é relativamente plano, o solo predominante é o Latossolo Vermelho e a produtividade florestal é de cerca de $55,0 \mathrm{~m}^{3} \mathrm{ha}^{-1} \mathrm{ano}^{-1}$. De acordo com a classificação de Köeppen, a região de Capão Bonito possui predominância dos tipos climáticos Cfa - subtropical, sem estação seca e com temperatura do mês mais quente superior a $22^{\circ} \mathrm{C} \mathrm{e} \mathrm{Cfb}$ - subtropical, sem estação seca, mas com temperatura do mês mais quente inferior a $22^{\circ} \mathrm{C}$. A variação da precipitação média 
anual gira entre 1.200 e $1.600 \mathrm{~mm}$, estando o déficit hídrico na faixa de 40 a $70 \mathrm{~mm}$ e temperatura média anual de $19,1^{\circ} \mathrm{C}$.

O povoamento florestal na área de estudo era do híbrido Eucalyptus urophylla X Eucalyptus grandis com idade de 7 anos, espaçamento de $3 \times 3$ m e estoque médio de madeira com casca de 404,2 $\mathrm{m}^{3} \mathrm{ha}^{-1}$, em regime de primeiro corte, com a finalidade de produzir matériaprima para celulose.

\subsection{Sistema de colheita}

Na colheita da madeira, o sistema utilizado era de árvores inteiras com o processamento da madeira em forma de cavacos na margem do talhão.

A operação tinha a seguinte sequência (Figura 1): As árvores eram abatidas utilizando tratores florestais fellerbunchers (marca Timberjack, modelo 608S; e John Deere, modelo 453G) e dispostas em feixes direcionados para a margem do talhão. Os feixes de madeira eram arrastados pelo trator florestal clambunk skidder (marca Timberjack modelo 1710D) e posteriormente transformados em cavacos pelo picador (marca Peterson Pacific, modelo 5000G).

\subsection{Especificação da máquina de extração}

O trator florestal arrastador clambunk skidder (Figura 2), da marca Timberjack, modelo 1710D, com três anos de uso, possuía motor John Deere 6081HT de seis cilindros, potência de $160 \mathrm{~kW}$ e sistema de transmissão hidrostática. Sua massa era de $17.000 \mathrm{~kg}$, com $10.100 \mathrm{~mm}$ de comprimento, $2.990 \mathrm{~mm}$ de largura e altura de $3.900 \mathrm{~mm}$. A capacidade de seu tanque de combustível era de 190 L de óleo diesel,



Figura 1 - Fluxograma do sistema de árvores inteiras. Figure 1 - Flowchart of the full-tree harvesting system. com consumo médio da ordem de $26 \mathrm{~L} \mathrm{he}^{-1}$ de operação; do tanque de óleo hidráulico, de 175 L; e de carga da máquina, de $14.000 \mathrm{~kg}$.

\subsection{Produtividade}

Para determinação da produtividade, foi utilizada a metodologia do estudo de tempos e movimentos, em nível do elemento do ciclo de trabalho.

\subsubsection{Estudo de tempos e movimentos}

\subsubsection{Ciclo de trabalho}

Os elementos componentes do ciclo operacional do clambunk skidder foram:

- Viagem sem carga: iniciava no momento em que a máquina começava a se locomover da margem do talhão em direção aos feixes de madeira dispostos no interior do talhão e terminava no instante em que se aproximava do feixe a ser arrastado, realizando a manobra.

- Manobra para o carregamento: começava no momento em que o trator chegava próximo do feixe a ser arrastado e começava a fazer a manobra, posicionando-se diante dos feixes para o carregamento, e terminava no instante em que era acionada a abertura das garras da pinça para o carregamento.

- Carregamento: iniciava quando o clambunk skidder estava posicionado diante dos feixes e acionava a abertura das garras da pinça, terminando quando o feixe estava seguro pela pinça.

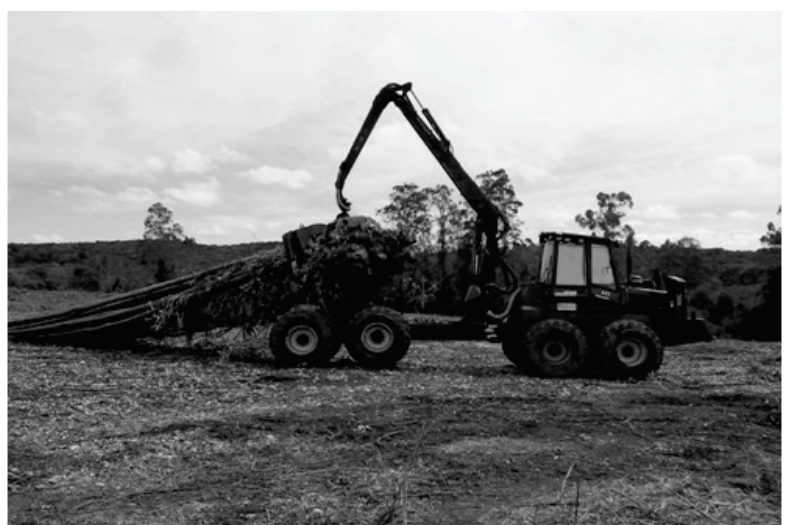

Figura 2 - Extração de madeira de eucalipto com clambunk Skidder,Timberjack, modelo1710D.

Figure 2 - Extraction of eucalyptus wood with clambunk Skidder, Timberjack, model 1710D.

Revista Árvore, Viçosa-MG, v.37, n.3, p.511-518, 2013 
- Deslocamento entre feixes: iniciava no momento em que a máquina se deslocava para um próximo feixe e terminava quando o trator iniciava a nova manobra para carregamento.

- Extração (arraste): tinha início quando a máquina começava a se deslocar com a carga e terminava quando chegava próximo aos feixes na margem do talhão.

- Descarregamento: iniciava no momento que, ao se aproximar das pilhas na margem da estrada, o operador manobrava e posicionava o feixe na pilha e terminava quando o feixe era liberado da pinça.

- Manobra na estrada: ocorria após o descarregamento da madeira, quando a máquina era posicionada para repetir o ciclo, e terminava quando era iniciada uma nova viagem sem carga.

- Deslocamento do "Over" (resíduo do tronco, grandes galhos e rejeitos não picados): tinha início no momento que o acúmulo do "Over", decorrente do corte da madeira em cavacos, começava a prejudicar o trânsito da máquina. Deslocava-se o “Over" das proximidades do picador, liberando a área para o descarregamento e manobras.

- Deslocamento de resíduo: iniciava assim que a deposição do resíduo, constituído, principalmente, por casca, folhas e pequenos galhos das árvores, começava a influenciar no deslocamento da máquina. Deslocava-se o resíduo das proximidades do picador, liberando a área para o descarregamento e manobras.

- Interrupções: foram classificadas em interrupções operacionais, interrupções mecânicas, espera de processamento da madeira pelo picador, espera pelo fim do ciclo de outro clambunk e espera para ajeitar feixes no campo.

\subsubsection{Medição dos tempos dos elementos de ciclo}

No estudo do ciclo operacional do clambunk skidder foi utilizado o método de tempo contínuo. Previamente foi realizado um estudo-piloto, no qual foram feitas cinco medidas de tempo do ciclo operacional para cada classe de distância, a fim de se estabelecer estatisticamente o número de observações necessárias. O número necessário de observações com erro relativo igual a $\pm 5 \%$ e $95 \%$ de confiança foi igual a 39, determinado utilizando-se a equação 1 (BARNES, 1977).

$$
N=\left(40 \frac{\sqrt{n \sum x^{2}-\left(\sum x\right)^{2}}}{\sum x}\right)^{2}
$$

em que:

$\mathrm{N}$ = número necessário de observações;

$\mathrm{n}=$ número de observações da amostra; e

$\mathrm{x}=$ valor de um elemento.

\subsubsection{Disponibilidade mecânica e eficiência operacional}

Disponibilidade da mecânica é a porcentagem do tempo trabalhado programado, em que a máquina não está em conserto ou manutenção, ou seja, é a porcentagem de tempo trabalhado programado, em que a máquina está mecanicamente apta a realizar trabalho produtivo, de acordo com a equação 2 . A eficiência operacional consiste na porcentagem de tempo realmente trabalhada, relacionando-se com o tempo total programado para o trabalho, conforme a equação 3 (BERARD, 1970). Adisponibilidade mecânica e a eficiência operacional foram calculadas utilizando-se os dados coletados durante o estudo (equações 2 e 3 ).

$$
\begin{gathered}
D M=\frac{H T P-H I M}{(H T P)} \times 100 \\
E O=\frac{H T E}{(H T P)} \times 100
\end{gathered}
$$

em que:

$\mathrm{DM}=$ grau de disponibilidade mecânica (\%);

HTP = tempo de trabalho programado (horas);

HIM = tempo de interrupção mecânica (horas);

$\mathrm{EO}=$ eficiência operacional $(\%)$; e

HTE $=$ tempo de trabalho efetivo (horas).

\subsubsection{Fatores influentes na produtividade do clambunk} skidder

São vários os fatores que influenciam na produtividade do clambunk skidder. Nesta pesquisa, somente a variável distância foi selecionada, dada a sua influência no sistema produtivo. A distância de extração, em metros, percorrida pelo clambunk entre a base do feixe formado durante a operação de abate 
e a praça de cavaqueamento foi medida com o computador de bordo da máquina. Foram consideradas distâncias entre $100 \mathrm{~m}$ e $370 \mathrm{~m}$.

Arelação entre distância e produtividade foi verificada utilizando-se a metodologia de análise de regressão. O coeficiente de determinção foi utilizado para verificar quanto da variabilidade total dos dados de produtividade era explicada pela varável distância.

\subsubsection{Avaliação da produtividade}

Na determinação da produtividade operacional, foi utilizada a equação 4 (MOREIRA et al., 2004).

em que: $\operatorname{Prod}\left(m^{3} c c h e^{-1}\right)=\frac{(N A \times V A)}{H E}$

Prod $=$ produtividade operacional, expressa $\mathrm{em} \mathrm{m}^{3} \mathrm{de}$ madeira com casca por tempo de trabalho efetivo;

$\mathrm{NA}=$ número de árvores obtido em um censo realizado a priori;

$\mathrm{VA}=$ volume médio por árvore, determinado a partir de análise do inventário $\left(\mathrm{m}^{3} \mathrm{cc}\right)$; e

$\mathrm{HE}=$ tempo de trabalho efetivo (horas).

\subsection{Avaliação de custos}

\subsubsection{Custo operacional do clambunk skidder}

A determinação do custo operacional foi adaptada da metodologia da FAO (1974), sendo os custos divididos em custos fixos, variáveis, administrativos e de pessoal, expressos em reais por hora efetiva de trabalho $\left(\mathrm{R} \$ \mathrm{he}^{-1}\right)$. Para realizar a estimativa do custo operacional, foi empregada a taxa de juros de $12 \%$ ao ano, o valor de revenda da máquina de $10 \%$ e a taxa de administração de $10 \%$ dos custos totais.

\subsubsection{Custo fixo $\left(\mathbf{R} \$ h^{-1}\right)$}

Este custo foi composto dos seguintes itens: depreciação linear, juros e seguros, em reais por hora efetiva de trabalho.

a) Depreciação: utilizada para recuperação do investimento original em uma máquina durante sua vida útil. O método utilizado foi o de depreciação linear simples.

b) Juros e seguros: utilizados para obter remuneração do capital investido a uma taxa de juros correspondente ao custo de oportunidade em que seria aplicado esse capital.

\subsubsection{Custo variável $\left(R \$ h^{-1}\right)$}

O custo variável, expresso em reais por hora efetiva de trabalho, foi composto dos seguintes itens, que se alteram diretamente com a utilização do maquinário.

a) Custo de combustível: calculado em função do consumo de óleo diesel.

b) Custo de lubrificantes e graxas: estimado em $25 \%$ do custo de combustível.

c) Custo do óleo hidráulico: estimado em $50 \%$ do custo de combustível.

d) Custo do conjunto pneus: calculado em função do preço dos pneus, do número de pneus utilizados e da sua vida útil.

e) Custo com manutenção e reparos: estimado em $80 \%$ da depreciação da máquina.

\subsubsection{Custo de administração ( $\left.\mathbf{R} \$ h^{-1}\right)$}

O custo de administração correspondeu aos custos com trabalhos administrativos de escritório e de supervisão das atividades de campo.

\subsubsection{Custo de pessoal operacional $\left(R \$ \mathrm{he}^{-1}\right)$}

Este custo foi calculado com base no salário direto acrescido do custo com benefícios e encargos sociais ( $13^{\circ}$ salário, férias, seguro, cuidados médicos, alimentação, vestuário e outros).

\subsubsection{Custo operacional $\left(\mathrm{R} \$ \mathrm{he}^{-1}\right)$}

Este custo foi obtido pela soma dos custos fixo, variável, de pessoal e de administração, expressos em reais por hora efetiva de trabalho (he).

\subsubsection{Custo de produção do clambunk skidder}

O custo de produção desta máquina $\left(\mathrm{R} \$ \mathrm{~m}^{-3} \mathrm{cc}\right)$ foi determinado a partir da divisão dos custos operacionais $\left(\mathrm{R} \$ \mathrm{he}^{-1}\right)$ pela produtividade $\left(\mathrm{m}^{3} \mathrm{cc} \mathrm{he} \mathrm{C}^{-1}\right)$, de acordo com a equação 5 .

$$
C P r=\frac{C T}{\operatorname{Pr} o d}
$$

em que:

$\mathrm{CPr}=$ custo de produção $\left(\mathrm{R} \$ \mathrm{~m}^{-3} \mathrm{cc}\right)$; 
$\mathrm{CT}=$ custo operacional $\left(\mathrm{R} \$ \mathrm{he}^{-1}\right) ; \mathrm{e}$

Prod $=$ produtividade $\left(\mathrm{m}^{3} \mathrm{cc} \mathrm{he} \mathrm{e}^{-1}\right)$.

\section{RESULTADOS}

\subsection{Produtividade}

\subsubsection{Ciclo de trabalho do clambunk skidder}

Na Tabela 1 estão apresentados os valores de tempo médio de 39 ciclos e a porcentagem dos elementos do ciclo operacional do clambunk skidder. O tempo total do ciclo operacional foi de $18,39 \mathrm{~min}$, sendo $14,40 \mathrm{~min}$ de tempo efetivo e 3,99 $\mathrm{min}$ de interrupções.

A principal interrupção ocorrida no ciclo operacional foi a espera de processamento da madeira pelo picador, com $4,41 \%$. As interrupções mecânicas corresponderam a $11,41 \%$ e as operacionais, a $3,43 \%$ do tempo total.

\subsubsection{Eficiência Operacional e Disponibilidade Mecânica}

A eficiência operacional obtida durante o estudo foi de $78,30 \%$, enquanto a disponibilidade mecânica, de $87,38 \%$.

\subsubsection{Produtividade operacional}

A produtividade operacional média do clambunk skidder foi de $80,25 \mathrm{~m}^{3} \mathrm{cc} \mathrm{he}^{-1} \mathrm{em}$ um povoamento com volume médio de $0,39 \mathrm{~m}^{3} \mathrm{cc}$ árvore ${ }^{-1}$, uma distância média de extração igual a 241,23 m e um volume de carga médio de $18,06 \mathrm{~m}^{3}$. A equação 6 , significativa $\left(\mathrm{p}<0,05 ; \mathrm{R}^{2}=71,45 \%\right)$, estimou a produtividade em função da distância de arraste. Na Figura 3 é apresentada a curva de estimativa da produtividade.

$$
P=116,005-0,148 \mathrm{D}
$$

$\mathrm{R}^{2}=71,45$

$\mathrm{p}<0,05$

em que:

$\mathrm{P}=$ produtividade estimada $\left(\mathrm{m}^{3} \mathrm{cc} \mathrm{he}^{-1}\right) ; \mathrm{e}$

$\mathrm{D}=$ distância de arraste $(\mathrm{m})$.

\subsection{Custos}

\subsubsection{Estimativa do custo operacional da máquina}

O custo operacional obtido foi igual a $\mathrm{R} \$ 377,30$ por hora efetiva de trabalho (he). Os custos variáveis corresponderam a 76,64\% do custo operacional, enquanto os custos fixos e os de adminstração e pessoal, a 14,27\% e $9,09 \%$ do custo total, respectivamente.

Tabela 1 - Elementos do ciclo operacional do clambunk Skidder, tempo médio consumido e porcentagem dos tempos. Table 1 -Elements of the clambunk operational cycle, average time consumed and time percentage.

\begin{tabular}{|c|c|c|}
\hline Elementos do ciclo operacional & Tempo médio (min) & Porcentagem do tempo médio total $(\%)$ \\
\hline \multicolumn{3}{|l|}{ Trabalho efetivo: } \\
\hline Viagem sem carga & 2,22 & 12,07 \\
\hline Manobra para carregamento & 0,29 & 1,57 \\
\hline Deslocamento entre feixes & 1,38 & 7,50 \\
\hline Carregamento & 4,46 & 24,22 \\
\hline Viagem com carga (arraste) & 2,94 & 15,95 \\
\hline Descarregamento & 0,32 & 1,74 \\
\hline Manobra na estrada & 0,37 & 2,07 \\
\hline Deslocamento de resíduo & 0,10 & 0,54 \\
\hline Deslocamento de over & 2,32 & 12,64 \\
\hline Subtotal efetivo & 14,40 & 78,30 \\
\hline \multicolumn{3}{|l|}{ Interrupções: } \\
\hline Espera de processamento da madeira pelo picador & 0,81 & 4,41 \\
\hline Espera pelo fim do ciclo de outro clambunk & 0,33 & 1,79 \\
\hline Espera para ajeitar feixes no campo & 0,14 & 0,76 \\
\hline Interrupções operacionais & 0,63 & 3,43 \\
\hline Interrupções mecânicas & 2,08 & 11,31 \\
\hline Subtotal interrupções & 3,99 & 21,70 \\
\hline Total & 18,39 & 100,00 \\
\hline
\end{tabular}

Revista Árvore, Viçosa-MG, v.37, n.3, p.511-518, 2013 


\subsubsection{Custo de extração}

O custo médio de extração do clambunk skidder foi igual a $\mathrm{R} \$ 4,91 \mathrm{~m}^{-3} \mathrm{cc}$ para uma distância média de extração de 241,23 m e para um povoamento com volume médio de $0,39 \mathrm{~m}^{3} \mathrm{cc}_{\text {árvore }}{ }^{-1}$. Este custo variou de $\mathrm{R} \$ 3,14$ a $\mathrm{R} \$ 6,93 \mathrm{~m}^{-3} \mathrm{cc}$, para distâncias de extração de $106 \mathrm{~m}$ e 366 m, respectivamente. Na Figura 4 é apresentada a relação entre a produtividade e o custo de extração.

\section{DISCUSSÃO}

A metodologia empregada e a análise dos resultados permitiram que a discussão a seguir possuísse argumentação lógica e com sustentação científica.

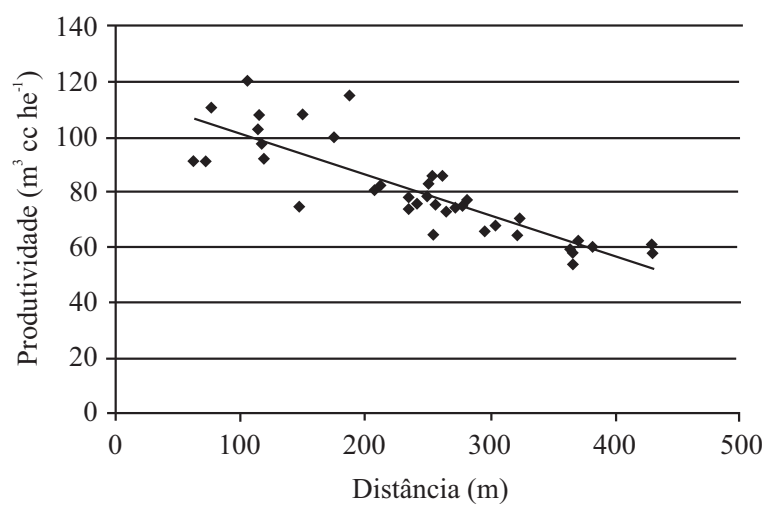

Figura 3 - Estimativa da produtividade do clambunk em função da distância de araste.

Figure 3 - Productivity estimation as a function of skidding distance.



Figura 4 - Comportamento do custo de extração de madeira em função da produtividade.

Figure 4 - Clambunk eucalyptus skidding cost as a function of productivity.
Os elementos que consumiram maior parte do tempo foram o carregamento e a viagem com carga. Por isso, a distância de extração e a organização do carregamento são variáveis importantes no planejamento do trabalho. Os tempos de interrupções, principalmente os de espera, são variáveis que podem ser eliminadas do processo, contribuindo para a melhoria da produtividade. Ledoux (2010) encontrou resultados semelhantes ao deste trabalho, e Spinelli e Visser (2009), analisando dados de estudo de tempo de máquinas florestais, indicaram que as interrupções variaram de forma significativa não só pelos tipos de máquina, mas pelas características dos povoamentos florestais e variáveis do terreno.

No sistema estudado houve influência significativa $(\mathrm{p}<0,05)$ da distância de arraste na produtividade do clambunk. O coeficiente de determinação indicou que aproximadamente $71 \%$ da variação na produtividade pode ser explicada pela variação na distância de arraste.

A análise de custos operacionais indicou que os mais importantes, em ordem decrescente, foram os custos variáveis, os custos fixos e os de administração, com destaque para o custo variável com combustível. A depreciação foi o custo fixo mais elevado.

O custo de extração do clambunk skidder foi influenciado pela distância de extração. Para distâncias entre aproximadamente 100 e 360 m, esse custo variou em mais de $140 \%$.

Oliveira et al. (2006), avaliando técnica e economicamente a extração com clambunk, encontraram custo de extração menor na classe de distância de 0 a $50 \mathrm{~m}$ em uma floresta com volume de $350 \mathrm{~m}^{3} \mathrm{ha}^{-1}$ e na declividade relativamente de 0 a $9^{\circ}$. Também, concluíram que a variável distância explicou significativamente a variação na produtividade.

A eficiência operacional e a disponibilidade mecânica, calculadas para o período de estudo, foram maiores que aquelas calculadas com dados coletados em longos períodos. Neste caso, com dados coletados apropriadamente haverá maior quantidade de dados do histórico da máquina, e a eficiência operacional e disponibilidade mecânicas serão mais representativas.

Revista Árvore, Viçosa-MG, v.37, n.3, p.511-518, 2013 


\section{CONCLUSÕES}

O planejamento da extração de madeira deve ter atenção especial às etapas de carregamento e viagem com carga, para que seja possível diminuir o tempo do ciclo de trabalho do clambunk. Os tempos de interruções variaram em cada ciclo e também poderiam ser reduzidos, eliminando-se tempos de espera.

À medida que aumenta a distância de extração, a produtividade diminui de maneira significativa.

Os custos variáveis, em especial o custo do combustível, foram os mais significativos. O custo de extração pode ser diminuído planejando-se adequadamente a distância de arraste e manejando interrupções evitáveis, para que o tempo de extração e os gastos com combustíveis e lubrificantes sejam menores.

A disponibilidade mecânica do clambunk foi alta devido à baixa incidência de interrupções para manutenção durante o período do estudo. A eficiência operacional do clambunk no período de estudo foi alta em razão do reduzido tempo de interrupções diversas encontradas na amostra estudada.

\section{AGRADECIMENTOS}

Ao Conselho Nacional de Desenvolvimento Científico e Tecnológico-CNPq, pelo suporte financeiro, pela bolsa de iniciação científica e de produtividade em pesquisa; e à Fundação de Amparo à Pesquisa do Estado de Minas Gerais - FAPEMIG, pelo suporte financeiro, na forma de bolsas de iniciação científica e do programa do pesquisador mineiro.

\section{REFERÊNCIAS}

ADEBAYO, A. B.;HAN, S. H.; JOHNSON, L.Productivity and cost of cut-to-length and wholetree harvesting in a mixed-conifer stand. Forest Products Journal, v.57, n.6, p. 59-68, 2007.

BARNES, R. M. Estudo de movimentos e tempos: projeto e medida do trabalho. São Paulo: Blucher, 1977.

BERARD, J. A. Standard definition for machine availability and utilization. Montreal: Canadian Pulp and Paper Associations, 1970.

Revista Árvore, Viçosa-MG, v.37, n.3, p.511-518, 2013
FAO.Logging and log transport in man-made forests in developing countries. Roma: 1974.

GOYCHUK, D. et al. The effect of timber harvesting guidelines on felling and skidding productivity in Northern Minnesota. Forest Science, v.57, n.5, p.393-407, 2011.

LeDOUX, C. B. Mechanized systems for harvesting eastern hardwoods. Newtown Square: U.S. Department of Agriculture, Forest Service, Northern Research Station, 2010. 13p. (Gen.Tech. Rep. NRS-69).

MACHADO, C. C. Exploração florestal, 6. Viçosa, MG: Universidade Federal de Viçosa, Imprensa Universitária, 1989. 34 p.

MOREIRA, F. M. T. et al. Avaliação operacional e econômica do "feller-buncher" em dois subsistemas de colheita de florestas de eucalipto. Revista Árvore, v.28, n .2, p.199-205, 2004.

NURMINEN, T.; KORPUNEN, H.; UUSITALO, J. Time consumption analysis of the mechanized cut-to-length harvesting system. Silva Fennica, v. 40, n.2, p. 335-363, 2006.

OLIVEIRA, R. J. et al. Avaliação técnica e econômica da extração de madeira de eucalipto com clambunk skidder. Revista Árvore, v.30, n.2, p. 267-275, 2006.

SPINELLI, R.; VISSER, R.J.M. Analyzing and estimating delays in wood chipping operations. Biomass and Bioenergy, v.33, n.3, p.429-433, 2009.

VALVERDE, S. R. et al. Análise técnica e econômica do arraste com "Skidder" no sistema de colheita de árvores inteiras de eucalipto. Revista Árvore, v.20, n.1, p.101-109, 1996.

WESTER, F.; ELIASSON, L. Productivity in ûnal felling and thinning for acombined harvesterforwarder (Harwarder). International Journal of Forest Engineering, v.14, n.2, p.45-50, 2003. 